\title{
Mathematical Modeling of Toxicological Effects of Fire Gases
}

\author{
G. E. HARTZELL, D. N. PRIEST, and W. G. SWITZER \\ Department of Fire Technology \\ Southwest Research Institute
}

P.O. Drawer 28510

San Antonio, Texas 78284, USA

\section{ABSTRACT}

Research in combustion toxicology over the past few years has led to a reasonable understanding and even quantification of some of the effects of fire effluent toxicants and, with the availability of a modest amount of both non-human primate and human exposure data, the combustion toxicologist is gaining increasing capability to assess and predict the toxicological effects of smoke inhalation.

This paper presents a mathematical approach, based on experimental data for $\mathrm{CO}, \mathrm{HCN}$ and $\mathrm{HCT}$, for the prediction of both incapacitating and lethal effects on rats exposed to these toxicants. Elementary examples are given for computer simulation of the development of toxic hazards in fires and comparisons are made with actual experimental results. These comparisons show that computer-predicted times to toxicological effects lie within the standard deviation of experimental mean values.

\section{INTRODUCTION}

From the wide variety of fire gases that may be generated, the toxicant gases may be separated into three basic classes: the asphyxiants or narcosis-producing toxicants; the irritants, which may be sensory or pu1monary; and those toxicants exhibiting other and unusual specific toxicities.

Although many asphyxiants may be produced by the combustion of materials, only carbon monoxide and hydrogen cyanide have been measured in fire effluents in sufficient concentrations to cause significant acute toxic effects. The toxicity of carbon monoxide is primarily due to its affinity for the hemoglobin in blood. Even partial conversion of hemoglobin to carboxyhemoglobin (COHb) reduces the oxygen-transport capability of the blood (anemic hypoxia), thereby resulting in a decreased supply of oxygen to body tissues. Hydrogen cyanide, a very rapidiy acting toxicant, does not combine appreciably with hemoglobin, but does bind with the trivalent ion of cytochrome oxidase in cellular mitochondria. The result is inhibition of the utilization of oxygen by cells (histotoxic hypoxia). Effects of the asphyxiant or narcosis-producing toxicants are dependent on the accumulated dose, i.e., both concentration and time of exposure, and increase in severity with increasing doses.

This work was supported under U.S. National Bureau of Standards Grant No. NB83NADA4015 and under Southwest Research Institute Internal Research Project No. 01-9316. 
From an extensive review of methodologies for assessment of the incapacitating effects of the narcotic fire gases, both with rats and with non-human primates, it has been concluded that rats appear to be sensitive to approximately the same range of accumulated doses as may be deemed potentially hazardous to human subjects [3]. Thus, for both carbon monoxide and hydrogen cyanide, the rat is expected to be a reasonably appropriate model for the development of methodology for estimating toxicological effects on humans.

Irritant effects, produced in essentially all fire gas atmospheres, are normally considered by combustion toxicologists as being of two types. These are sensory irritation, including irritation both of the eyes and of the upper respiratory tract, and pulmonary irritation. Most irritants produce signs and symptoms characteristic of both sensory and pulmonary irritation, however.

Airborne irritants enter the upper respiratory tract, where nerve receptors are stimulated causing characteristic physiological responses, including burning sensations in the nose, mouth and throat, along with secretion of mucus. Senosry effects are primarily related to the concentration of the irritant and do not normally increase in severity as the exposure time is increased. There is no evidence that sensory irritation, per se, is physically incapacitating, either to rodents or to primates. On the contrary, recent studies involving exposure of baboons to hydrogen ch Toride have shown that even massive concentrations (up to 17,000 ppm for 5 minutes) are not physically incapacitating and do not impair escape [4].

of potentially greater importance with both rodents and primates is that, following signs of initial sensory irritance, significant amounts of inhaled irritants are quickly taken into the lungs with the symptoms of pulmonary or lung irritation being exhibited. Tissue inflammation and damage, pulmonary edema and subsequent death often follow exposure to high concentrations, usually after 6 to 48 hours. UnTike sensory irritation, the effects of pulmonary irritation are related both to the concentration of the irritant and to the duration of the exposure.

In view of the importance of the common asphyxiants and also of pulmonary irritation, this study focused on the development of models for predicting the effects of $\mathrm{CO}, \mathrm{HCN}$ and $\mathrm{HCl}$ on rats. Only the post-exposure lethality of rats was felt significant for the $\mathrm{HCl}$ part of this study; however, both incapacitation (leg-flexion shock-avoidance) and withinexposure lethality were employed for $\mathrm{CO}$ and $\mathrm{HCN}$.

\section{MATHEMAT ICAL MODEL ING}

The basic concepts for toxicological modeling developed here involve the use of concentration-time relationships as a quantification of the "exposure dose" required to produce a given effect. From fundamental principles of toxicology, it has been demonstrated that concentration, mean exposure time to effect a given response, and percent of subjects responding are interrelated mathematically [6]. For example, a plot of mean exposure time required to effect a response as a function of concentration (Ct plot) a 1so represents the $E C_{50}$ (or $L C_{50}$ ) as a function of exposure time, or conversely, the $\mathrm{ET}_{50}$ (or $\mathrm{LT}_{50}^{50}$ ) as a function of concentration.

Typical concentration-time relationships are illustrated in Figure 1 for incapacitation and lethality of rats from exposure to carbon monoxide. From this figure, the "exposure dose" required to cause incapaci- 
tation can, for example, be obtained by multiplying the values of any pair of coordinates; e.g., $2000 \mathrm{ppm} \times 20 \mathrm{~min}=40,000 \mathrm{ppm}$-min "exposure dose." The "exposure dose" required to cause an effect for a given toxicant is relatively constant (Haber's Rule) over the curved portion of the plot. For many purposes, the relative constancy of the ct product can be used as a first approximation for estimation of the time-to-effect for a desired exposure concentration. However, for purposes of developing mathematical models having predictive value, further refinements must be made to take into account the fact that the ct product "exposure dose" decreases with increasing concentration and may actually cover a two- to three-fold range.

\section{CARBON MONOXIDE INTOXICATION OF RATS}

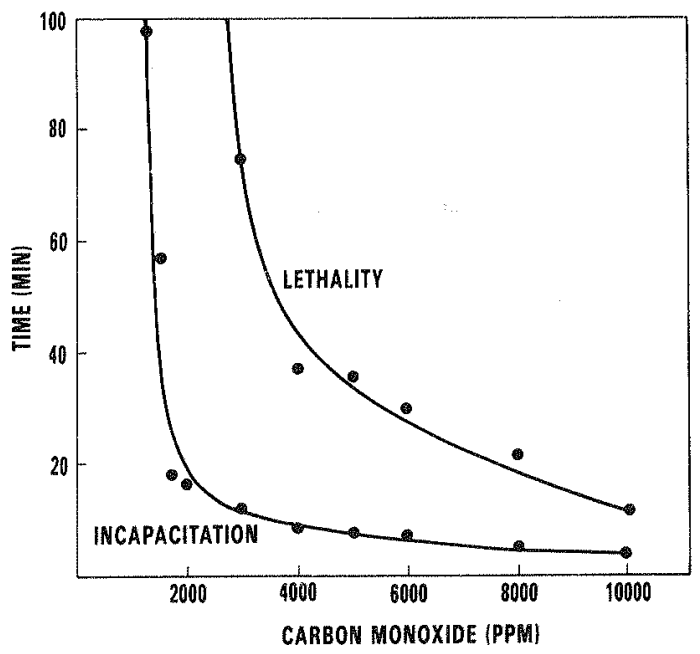

\section{FIGURE 1.}

Specific ct "exposure doses" required to produce a given toxicological effect for a particular concentration may be determined as follows:

From the expression for Haber's Rule,

$c t=k$,

simple rearrangement of terms produces

$c=k\left(\frac{1}{t}\right)$.

This is a linear equation, indicating that a plot of concentration against the reciprocal of time should be a straight line with the slope being $k$. Figures 2 and 3 , using mean times-to-effect for exposure of rats to carbon 
monoxide, show that the relationship is, indeed, linear. Similar linear relationships between concentration and the reciprocal of time-to-effect are shown in Figures 4 and 5 for incapacitation and death of rats due to exposure to hydrogen cyanide.

In the case of lethality of rats from hydrogen chloride, time of exposure required to effect 50 percent post-exposure lethality, rather than time to death, must be used. Therefore, $L C_{50}$ values, determined for a series of different exposure times, are plotted as a function of the reciprocal of exposure time as shown in Figure 6 . In contrast to Figures 2 through 5, which show mean times-to-effect for CO and HCN exposures, Figure 6 reflects the time of exposure required to cause 50-percent postexposure lethality for any concentration of $\mathrm{HCl}$.

\section{CARBON MONOXIDE INTOXICATION OF RATS}

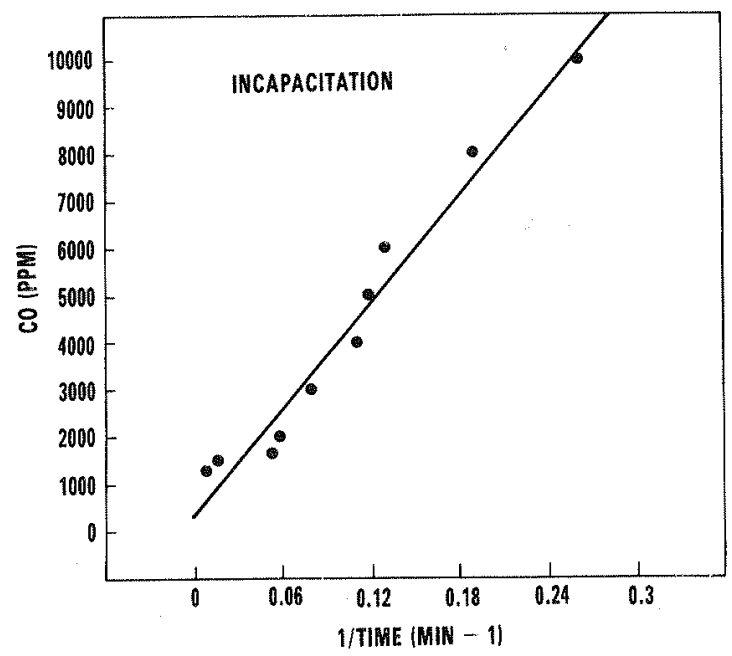

FIGURE 2. 
CARBON MONOXIDE INTOXICATION OF RATS

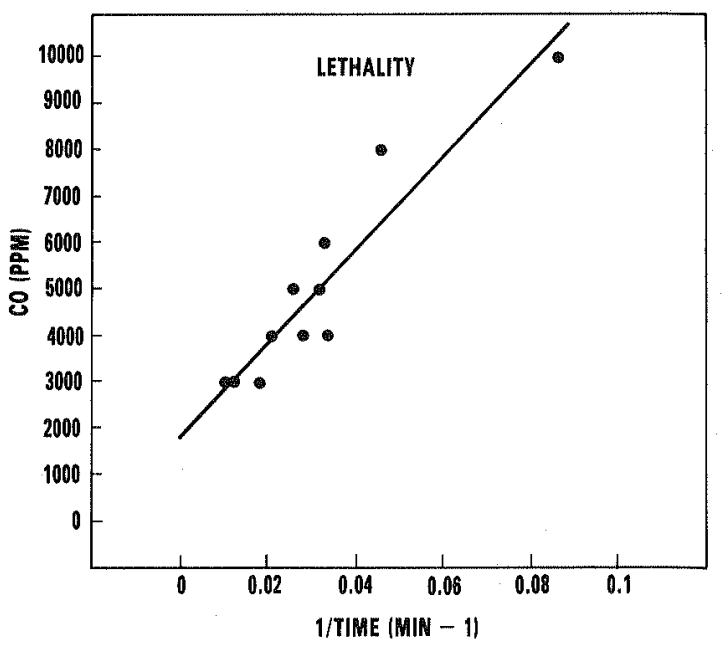

FIGURE 3.

HYDROGEN CYANIDE INTOXICATION OF RATS

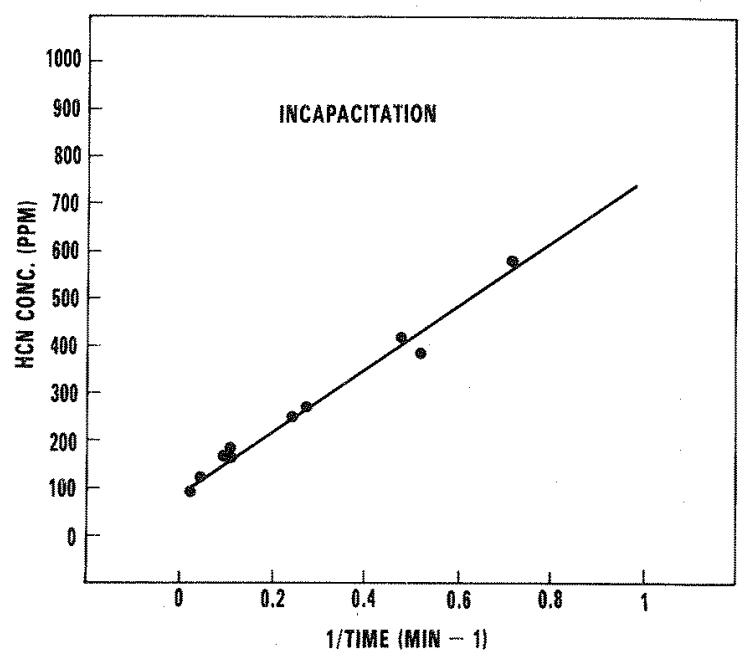

FIGURE 4. 
HYDROGEN CYANIDE INTOXICATION OF RATS

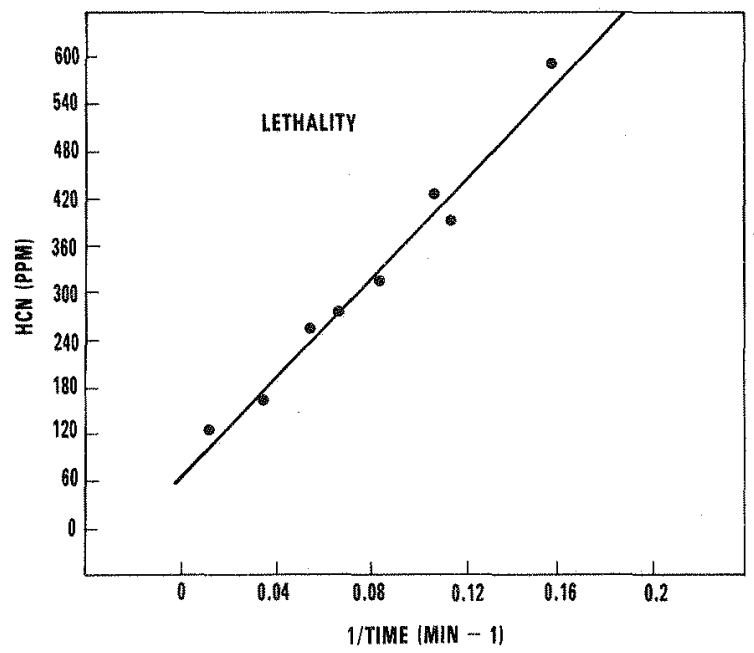

FIGURE 5.

POST-EXPOSURE LETHALITY OF RATS FROM EXPOSURE TO HYDROGEN CHLORIDE

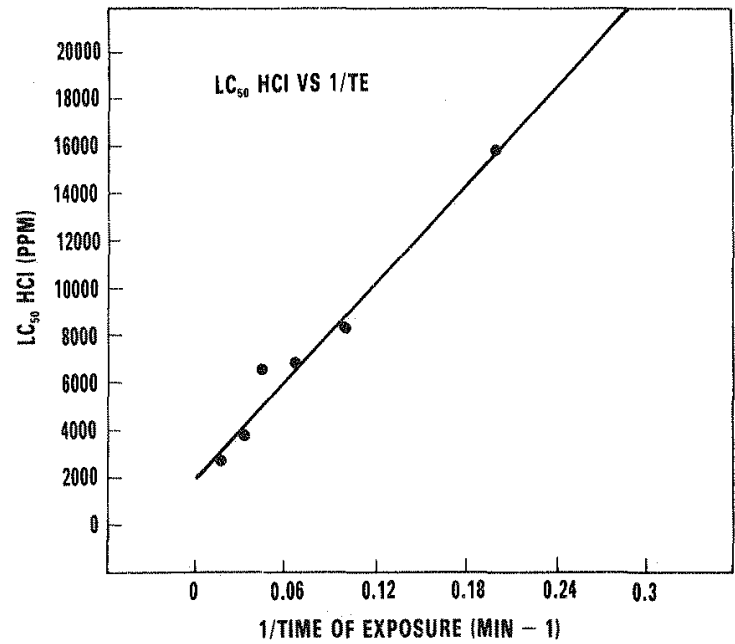

FIGURE 6 . 
Since the plots of $1 / t$ against concentration do not pass through the origin, equation (2) must be modified to include b, the $y$-axis intercept.

$c=k\left(\frac{1}{t}\right)+b$

Equation (3) can be used directly for calculation of the $E C_{50}$ or $L C_{50}$ for any desired time of exposure once the constants have been determined. From equation (3) can also be derived an expression for the ct product "exposure dose" as a function of the concentration,

$c t=k\left(\frac{C}{C-b}\right)$,

where $K$ and $b$ are determined from the linear plot of experimental data. Equation (4) enables calculation of the ct product "exposure dose" of the toxicant required to produce a given effect when present at any concentration. The slope $K$ of the plot of concentration vs. 1/t represents the minimum value of the "exposure dose" required to effect a 50-percent response at sufficiently high concentrations such that the term $(C / C-b)$ approaches unity. Under these conditions, Haber's Rule (i.e., $C t=K$ ) would be valid. The $y$-axis intercept, $b$, would thus appear to be a measure of deviation from Haber's Rule at relatively low concentrations of a toxicant.

In Table $I$ are presented the values of $K$ and $b$ for both incapacitation and lethality for $\mathrm{CO}$ and $\mathrm{HCN}$, and post-exposure lethality for HCl. Examples of calculated $E_{50}$ 's and $E(C t)_{50}$ 's for 15- and 30-minute exposures of rats are also shown. 'Data in Table 1 were derived from populations of 138 and 78 rats for incapacitation and lethality due to $\mathrm{CO}$, populations of 70 and 46 rats for incapacitation and lethality due to $H C N$ and a population of 180 rats for post-exposure lethality due to $\mathrm{HCl}$ exposures.

TABLE I. Tabulation and use of modeling constants

\begin{tabular}{|c|c|c|c|c|c|c|}
\hline & $k$ & b & $\begin{array}{r}E C_{50} \\
15-\min \\
(\mathrm{ppm}) \\
\end{array}$ & $\begin{array}{r}E C_{50} \\
30-m^{-m i n} \\
(\mathrm{ppm}) \\
\end{array}$ & $\begin{array}{c}E(C t)_{50} \\
15-m i n \\
(p p m-m i n) \\
\end{array}$ & $\begin{array}{r}E(c t)_{50} \\
30-m i n \\
(p m-m i n) \\
\end{array}$ \\
\hline & \multicolumn{6}{|c|}{ INCAPAC ITATION } \\
\hline co & 36,509 & 233 & 2,667 & 1,450 & 40,004 & 43,499 \\
\hline \multirow[t]{2}{*}{ HCN } & 698 & 92 & 139 & 115 & 2,078 & 3,458 \\
\hline & \multicolumn{6}{|c|}{ LETHALITY } \\
\hline CO & 102,874 & 1,778 & 8,636 & 5,207 & 129,544 & 156,214 \\
\hline HCN & 3,130 & 66 & 275 & 170 & 4,120 & 5,110 \\
\hline $\mathrm{HCl}$ & & & & & & \\
\hline (post-exposure) & 69,299 & 1,935 & 6,555 & 4,245 & 98,324 & 127,349 \\
\hline
\end{tabular}

A restriction on the direct application of simple concentration-time relationships is that they are appropriate only for modeling constant concentration or "square wave" toxicant exposures such as those from which they were obtained experimentally. Fires do not produce constant toxicant concentrations, but rather, produce changing (usuatiy increasing) concentrations. Mathematical models to predict effects from fire-generated toxicants must be able to accommodate varying concentrations which, in turn, involve varying $C t$ "exposure doses" required to effect incapacitation and/or death. 
In Figure 7 is illustrated conceptually how this may be accomplished. From a plot. of toxicant concentration as a function of time, incremental "doses" ( $C \times \Delta t)$ are calculated and related to the specific $c t$ "exposure dose" required to produce the given toxicological effect at the particular incremental concentration. Thus, a "fractional effective dose" (FED) is calculated for each small time interval. Continuous summation of these "fractional effective doses" is carried out and the time at which this sum becomes unity represents the time of greatest probability of occurrence of the toxicological effect in 50 percent of the exposed subjects. (As with all statistical data, there is a probability distribution for the predicted time-to-effect.)

\section{MODELING OF TOXICOLOGICAL EFFECTS OF FIRE GASES}

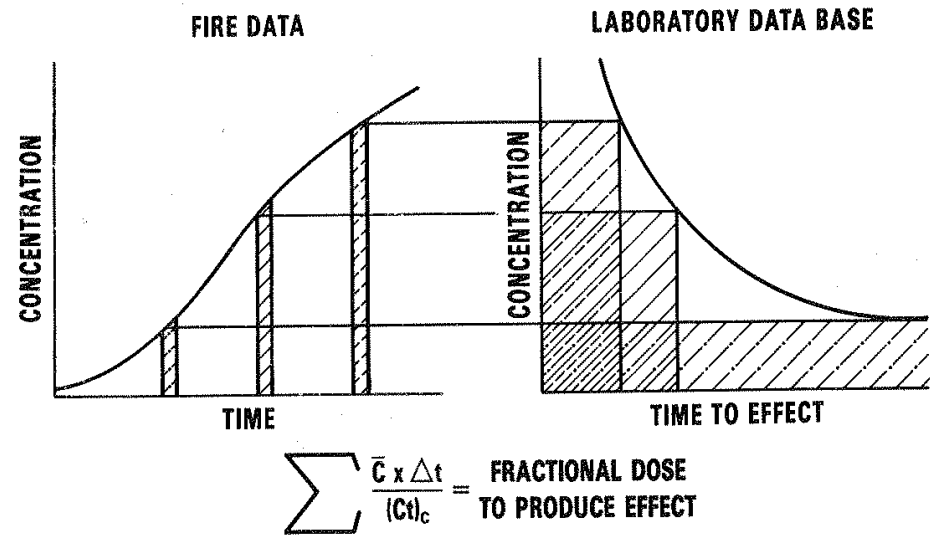

EFFECT OCCURS AT TIME $t$ WHEN $\Sigma$ FRACTIONAL DOSES $=1$

FIGURE 7.

The laboratory data $c t$ curve illustrated in Figure 7 is presented for conceptual purposes only. In practice, the specific ct "exposure dose" corresponding to each incremental concentration is calculated from Equation (4).

The mathematical modeling concept illustrated in Figure 7 was tested for both incapacitation and lethality of rats exposed to increasing concentrations of carbon monoxide as might occur in a fire. The carbon monoxide concentration was ramped from 0 to about $9500 \mathrm{ppm}$ over a period of about 10 minutes, followed by maintaining the concentration at the maximum leve1. By continuous summation of fractional effective doses for each 15 seconds of exposure, computer projections estimated incapacitation of exposed rats should occur at 10.0 minutes, with death expected at $20.8 \mathrm{~min}$ - 
utes. Actual exposure of six rats to the ramped conditions yielded mean times of $10.2 \pm 1.9$ and $22.8 \pm 3.5$ minutes for incapacitation and death, respectively. The predicted times to both incapacitation and death were well within the standard deviations for the experimentally determined values.

Another test of the fractional effective dose model for carbon monoxide was conducted which employed a slower ramping profile for generation of the toxicant. The maximum $c 0$ concentration was attained at about 30 minutes. The computer-predicted time to incapacitation of 16.5 minutes was in excellent agreement with the experimental time of $17.2 \pm 1.5$ minutes determined using five rats. Death of the rats was predicted at $32.5 \mathrm{~min}$ utes. The experimentally determined mean time-to-death was $43.9 \pm$ 13.9 minutes, with two of the five rats dead by 33.0 minutes.

The model was also tested for hydrogen cyanide, with exposure of six rats to. HCN ramped from 0 to $195 \mathrm{ppm}$ over about 12 minutes, followed by maintaining that concentration throughout the remainder of the exposure. Incapacitation and death were predicted by computer for 13.8 minutes and 32.2 minutes, respectively. Experimentaliy, mean times-to-incapacitation and death were $13.8 \pm 2.3$ minutes and $36.8 \pm 11.9$ minutes, respectively. Three of the six rats had died by 32 minutes.

A second test of the FED model for hydrogen cyanide was conducted with the concentration of $\mathrm{HCN}$ ramped from 0 to 270 ppm over 30 minutes. The model predicted incapacitation and death for 22.8 minutes and 34.0 minutes, respectively. Mean times-to-incapacitation and death of the six rats exposed in the ramped test were $20.8 \pm 2.8$ minutes and $34.2 \pm 2.1$ minutes, respectively.

A further refinement of the FED model enabled estimation of the error expected in the projection of times to incapacitation and death for $\mathrm{CO}$ and HCN. This involved determination of the $K$ and $b$ modeling constants for concentration vs. $1 / t$ plots representing standard deviations of the original time-to-effect data. Analogous to the sumation of fractional effective doses for the projection of mean times-to-effect, summations were also carried out corresponding to $c t$ products representing standard deviations of the original laboratory data. A comparison of computerprojected and experimental data, including predicted errors and/or standard deviations is presented in Tables II and III. It can be seen that predicted and experimental ranges for times to effect are sufficiently comparable as to suggest that model predictions are as accurate as experimental determinations.

TABLE II. Comparison of FED mode' with experimental data for carbon monoxide

\begin{tabular}{|c|c|c|c|c|}
\hline & \multicolumn{2}{|c|}{$\begin{array}{c}\text { Incapacitation } \\
\text { (Minutes) }\end{array}$} & \multicolumn{2}{|c|}{$\begin{array}{l}\text { Lethality } \\
\text { (Minutes) }\end{array}$} \\
\hline & Mean & Range (S.D.) & Mean & Range (S,D.) \\
\hline FED Model & 10.0 & $7.5-11.0$ & 20.8 & $17.0-24.0$ \\
\hline Experimenta 1 & 10.2 & $8.3-12.1$ & 22.8 & $19.3-26.3$ \\
\hline FED Model & 16.5 & $12.2-18.2$ & 32.5 & $26.8-37.2$ \\
\hline Experimental & 17.2 & $15.7-18.7$ & 43.9 & $30.0-57.8$ \\
\hline
\end{tabular}


Table III. Comparison of FED model with experimental data for hydrogen cyanide

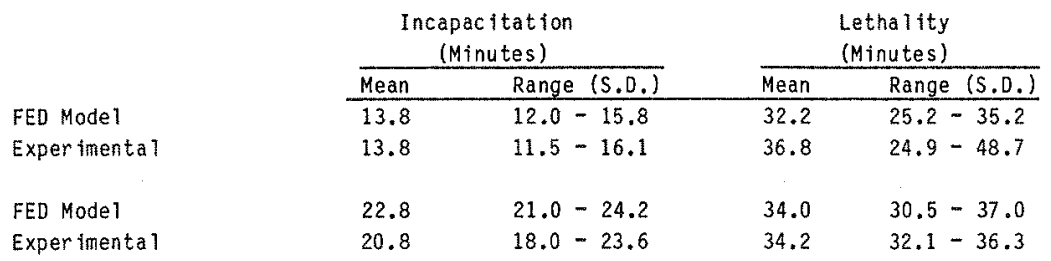

Validation of the fractional effective dose model for exposure of rats to $\mathrm{HCl}$ would be expected to be somewhat more difficult, since lethality occurs up to 14 days post-exposure and is due to indirect causes. Hydrogen chloride was ramped stepwise to a maximum concentration of about $6400 \mathrm{ppm}$. The FED summation reached a value of 0.87 at 28.5 minutes. The six exposed rats were then withdrawn and observed for up to 14 days, with the expectation that half should survive. Although all the subjects died with in the 14-day period, this result is not unusual for a single experiment. The confidence limits for experimental post-exposure $L_{50}{ }^{\prime} s$ of $\mathrm{HCl}$ are quite broad, due, in part, to wide animal response variability with post-exposure effects.

The validity of the FED model has thus far been tested onty for exposure of rats to single toxicants. However, with input data from appropriate Taboratory experiments, the model would be expected to be quite appropriate for combinations of toxicants. The initial success of this methodology is anticipated to open the way to the combustion toxicity testing of materials without the necessity of using laboratory animals on a routine basis. Furthermore, the estimation of toxicological effects of smoke inhalation by humans exposed in a fire would also appear to be feasible.

\section{REFERENCES}

1. Kaplan, H. L., Grand, A. F. and Hartze11, G. E., Combustion Toxicology: Principles and Test Methods, Technomic Publishing Company, Incorporated, Lancaster, Pennsylvania, 1-20, 1983.

2. Hartze17, G. E., Packham, S. C. and Switzer, W. G. "Toxic Products from Fires," Am. Ind. Hyg. Assoc. J., 44 (4), 248-255, 1983.

3. Kaplan, H. L. and Hartze11, G. E. "Modeling of Toxicological Effects of Fire Gases: I. Incapacitating Effects of Narcotic Fire Gases," J. Fire Sciences, Vo 7.2 , No. 4, 286-305, 1984.

4. Kaplan, H. L. et aT., "A Research Study of the Assessment Escape Impairment by Irritant Combustion Gases in Postcrash Aircraft Fires," Southwest Research Institute, Fina T Report, DOT/FAA/CT-84/16, U. S. Department of Transportation, Federal Aviation Administration, Atlantic City, New Jersey, 1984.

5. Kaplan, H. L., Southwest Research Institute, Unpublished Work.

6. Packham, S. C. and Hartze11, G. E., "Fundamentals of Combustion Toxicology in Fire Hazard Assessment," Journal of Testing and Evaluation, Volume 9, No. 6, 341-347, November 1982. 